\title{
Is the increased transmissibility of SARS-CoV-2 variants driven by within or outside-host processes?
}

\author{
Yehuda Arav $^{1}$, Eyal Fattal ${ }^{1}$, and Ziv Klausner ${ }^{1}$ \\ ${ }^{1}$ Israel Institute for Biological Research
}

December 29, 2021

\begin{abstract}
Understanding the factors that increase the transmissibility of the recently emerging variants of SARS-CoV-2 can aid in mitigating the COVID-19 pandemic. The enhanced transmissibility could be attributed to enhanced within-host processes, such as contagiousness (viral shedding by an infected individual) and infectivity (the probability of a susceptible individual to get infected), or outside-host processes, such as viral stability on surfaces and in the air. We utilized a mathematical model in order to theoretically analyze the specific mechanisms of virus transfer between an infected and susceptible individual. This allowed us to examine how the within-host or outside-host processes affect the overall viral transmission. Our analysis is based the available data on the Alpha, Epsilon and Delta variants as well as the currently emerging Omicron variant. We found that the higher transmissibility of the SARS-CoV-2 variants can be attributed only to within-host processes. Specifically, enhanced contagiousness drives the Delta variant transmissibility, while the Alpha, Epsilon and Omicron are characterized by an enhanced infectivity. Since outside-host processes have little contribution to the observed increase in the transmissibility, leading stricter hygienic and behavioral measures than those that were already applied are not expected to achieve a pronounced mitigating effect.
\end{abstract}

\section{Hosted file}

paper2021-variants_transmissibility.docx available at https://authorea.com/users/453042/ articles/550978-is-the-increased-transmissibility-of-sars-cov-2-variants-driven-bywithin-or-outside-host-processes 